\title{
NOTAS CARIOLÓGICAS SOBRE ALGUNAS COMPUESTAS DE ANDALUCÍA
}

\author{
Montserrat ARISTA, Salvador TALAVERA y Pedro Luis ORTIZ
}

RESUMEN. Notas cariológicas sobre algunas compuestas de Andalucía. En esta nota se presentan los números somáticos de 11 especies de la Fam. Compositae, ocho de ellas pertenecientes al género Centaurea. Se estudian también los cariogramas de Centaurea bombycina, C. sulfurea, Hedypnois ragadioloides y Hypochoeris salzmanniana. Los números cromosómicos encontrados en Centaurea son $2 \mathrm{n}=20,22,24$ y 32; en Hypochoeris y en Hedypnois $2 \mathrm{n}=8$, y en Carthamus lanatus subsp. baeticus $2 \mathrm{n}=44$.

Palabras clave. Cariología, Compositae, Centaurea, Hedypnois, Hypochoeris, Carthamus.

SUMMARY. Kariological notes on some Compositae from Andalusia. The chromosome numbers of 11 species of the family Compositae, eight of which beloging to the genus Centaurea, are reported in this paper. The caryograms of Centaurea bombycina, C. sulfurea, Hedypnois ragadioloides and Hypochoeris salzmanniana are also studied. The chromosome numbers found in Centaurea are $2 n=20,22,24$ and 32 ; in Hypochoeris and Hedypnois $2 \mathrm{n}=8$, and in Carthamus lanatus subsp. baeticus $2 \mathrm{n}=44$.

Key words. Karyology, Compositae, Centaurea, Hedypnois, Hypochoeris, Carthamus.

\section{INTRODUCCIÓN}

El estudio cariológico se ha realizado en meristemos radicales obtenidos de plantas recolectadas en el campo y cultivadas posteriormente en el invernadero de la Universidad de Sevilla. Los testigos se encuentran depositados en el herbario del Departamento de Biología Vegetal y Ecología de la Universidad de Sevilla (SEV). Las raíces se trataron con 8-hidroxiquinoleína $0,002 \mathrm{M}$ a $4^{\circ} \mathrm{C}$ durante $3 \mathrm{~h} 30^{\prime}$ y se fijaron en alcohol etílico con ácido acético glacial (3:1) durante $24 \mathrm{~h}$, realizándose la tinción siguiendo la técnica descrita por Snow (1963). Para caracterizar la morfología de los cromososmas se ha seguido la terminología propuesta por Levan et al. (1964), y para determinar el tamaño de los cromosomas y grados de asimetría de los cariotipos, la indicada por Stebbins (1938 y 1971, respectivamente).

Carthamus lanatus subsp. baeticus (Boiss. \& Reuter) Nyman

$2 n=44$

CÁDIZ: Punta Paloma. Arenales costeros, IV.1995, Arista, M., P. L. Ortiz y S. Talavera, 32/ 95, SEV 136155.

El número somático encontrado difiere del $2 n=64$ indicado por Khidir \& Knowles (1970) en material procedente de Portugal. Al parecer es el primer recuento de esta especie en material español.

Centaurea bombycina Boiss.

$2 \mathrm{n}=20$ (fig. 1.1, 2A)

MALAGA: Canillas de Albaida. Sierra Tejeda, 700 m, dolomías, 3.II.1995, Arista, M., P. L. Ortiz y S. Talavera, 15/95, SEV 136153.

El número encontrado difiere del $2 \mathrm{n}=18$ 
indicado por Fernández Morales (1974) en material de Granada. Sin embargo, podría ser coincidente con el $2 n=18+(0-2)$ B encontrado por Blanca López (1981) en material de Granada. Este es el primer recuento de esta especie, endémica de Málaga y Granada, en material procedente de Málaga.

El cariograma (fig. 2A) tiene la fórmula cromosómica $8 \mathrm{~m}+1 \mathrm{~m}-\mathrm{sm}+1 \mathrm{sm}$. El tipo de asimetría es $1 \mathrm{~A}$, y el tamaño aparente de los cromosmas de nuestro material osciló entre 4,4-7,2 $\mu \mathrm{m}$ (medianamente pequeños y medianamente grandes respectivamente).

\section{Centaurea clementei Boiss.}

$2 n=20$

MÁlagA: Puerto de las Pedrizas. El Colmenar, $730 \mathrm{~m}$. Terra rossa entre calcáreos. 2.II.95. Arista, M., M.C. Machado, P.L. Ortiz y S. Talavera, 5A, SEV 136152.

El número cromosómico encontrado coincide con los indicados por Fernández Morales (1974) y por Luque, Mejías y Díaz Lifante (1988), ambos en material de Cádiz. Así mismo coincide con el número gamético $n=10$, indicado por estos últimos.

Centaurea exarata Boiss. ex Coss.

$2 n=22$ (fig. 1.2 )

HUELVA: Hinojos. Casa forestal Cumbres Hermosas. IV.1995. S. Talavera. 38/3. SEV 136151.

El número somático hallado coincide con el encontrado por Valdés-Bermejo (1980) en material de Huelva, pero difiere del número cromosómico $2 \mathrm{n}=18$ y del gamético $\mathrm{n}=9$ indicado por Fernández Morales (1974) en plantas de Granada. Nuestro recuento confirma el de Valdés-Bermejo (1980).

\section{Centaurea melitensis L.}

\section{$2 \mathrm{n}=24$}

HUELVA: Hinojos. Casa forestal Cumbres Hermosas. Alcornocal. IV.1995. S. Talavera, 39/ 19, SEV 136150.

El número somático encontrado coincide con el hallado por diversos autores (véase Castroviejo y Valdés-Bermejo, 1991 y Pastor, 1992), pero difiere del número 2n=36 indicado por Chiappini (1954).

\section{Centaurea prolongoi Boiss.}

$2 n=20$ (fig. 1.3)

MÁlAGA: Ojén, $750 \mathrm{~m}$, Terra rossa. 3.II.1995. Arista, M., P. L. Ortiz y S. Talavera, 23/

\section{9, SEV 136154.}

El número cromosómico encontrado es coincidente con el hallado por Fernández Morales (1974) y con el de Valdés-Bermejo y Agudo Mata (1983), ambos en material procedente de Málaga.

\section{Centaurea sphaerocephala $\mathrm{L}$.}

$2 n=22$

CÁDIZ: Chiclana de la Frontera. Cabo Roche. Matorrales costeros sobre arenas. IV.1995. Arista, M., P. L. Ortiz y S. Talavera, 26/2, SEV 136149. Cabo de Trafalgar. El Faro. Arenales marítimos. IV.95. Arista, M., P. L. Ortiz y S. Talavera, 29/1, SEV 136156.

En las dos poblaciones estudiadas el $n^{\circ}$ cromosómico hallado fue $2 n=22$. Estos recuentos son coincidentes con el dado para este taxón (sub C. polyacantha Wild.) por Valdés (1973) y ValdésBermejo (1980) en plantas de Huelva, Gadella et al. (1966) y Gallego y Aparicio (1990) en material de Cádiz, Fernandes y Queirós (1971) y Guinochet \& Foissac (1962) en plantas de Portugal, y con el de Talavera et al. (1984) en material de Marruecos. Difiere sin embargo, del tetraploide $2 n=44$ encontrado por Guinochet \& Foissac (1962), Damboldt \& Matthäs (1975) y por Bartolo et al. (1977), así como del $2 \mathrm{n}=36$ indicado por Chiappini (1954).

\section{Centaurea sulfurea Willd}

2n=24 (fig. 1.4, 2B)

MÁlAGA: Puerto de las Pedrizas. El Colmenar, $730 \mathrm{~m}$. Terras rosas entre calcáreos. 2.II.95. Arista, M., M.C. Machado, P. L. Ortiz y S. Talavera, 3/95, SEV 136146.

El número somático encontrado coincide con los recuentos realizados sobre material de Granada y Málaga por diversos autores (véase Castroviejo y Valdés-Bermejo, 1991).

El cariograma (fig. 2B) tiene la fórmula cromosómica $8 m+2 s m+1 \mathrm{sm}^{\text {sal }}+1$ st. Según su simetría la denominación del cariotipo es $1 \mathrm{~A}$. El tamaño cromosómico aparente en la población estudiada osciló entre 2,6-5 $\mu \mathrm{m}$ (entre pequeños y medianamente pequeños).

\section{Centaurea uliginosa Brot.}

$$
2 n=32
$$

HUELVA: Almonte. Los Huertecillos. Suelo higroturboso con Ulex minor y Erica ciliaris, 


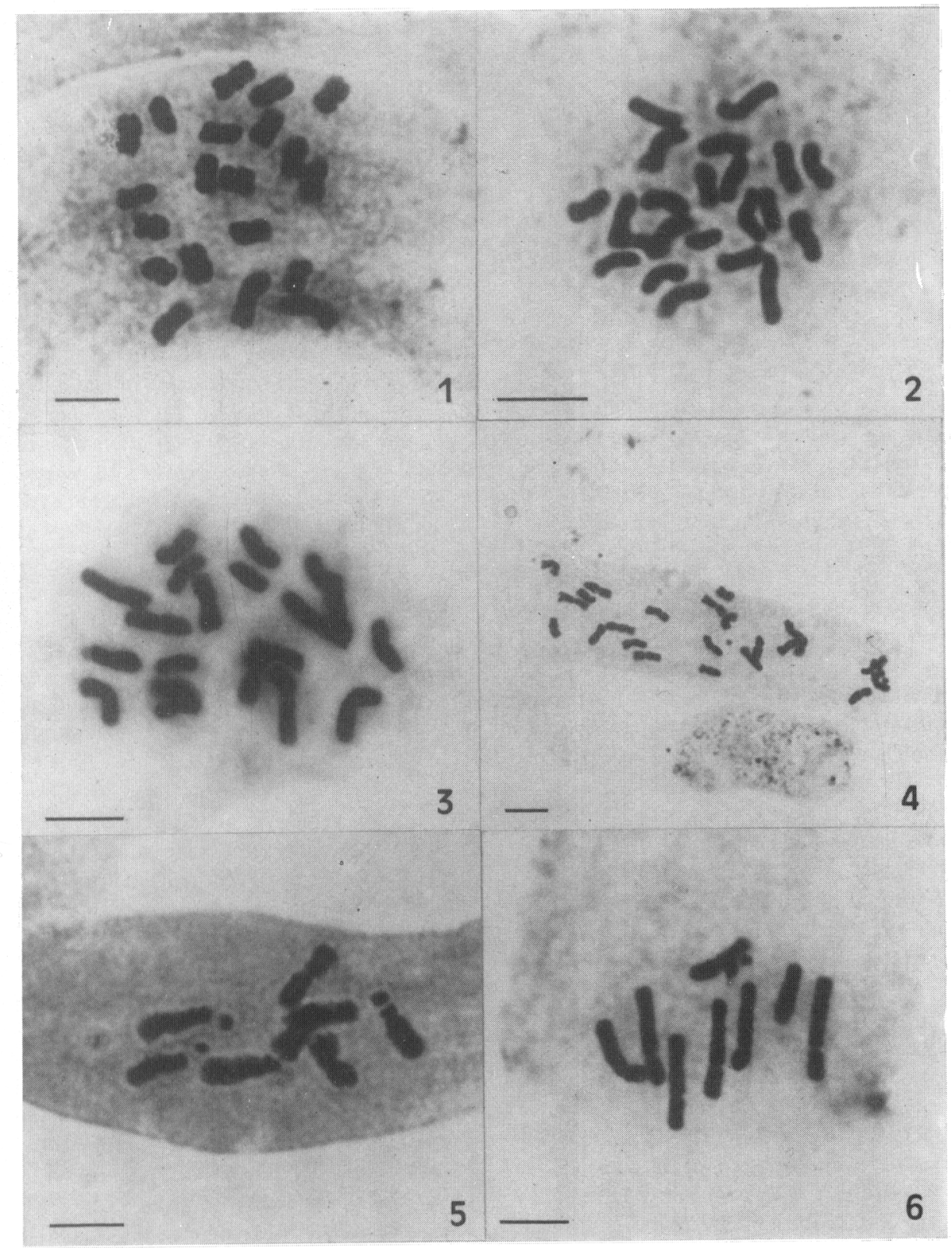

Figura 1. Metafase somática de (1) Centaurea bombycina, $2 \mathrm{n}=20$; (2) C. exarata, $2 \mathrm{n}=22 ;$ (3) C. prolongoi, $2 \mathrm{n}=20$; (4) C. sulfurea, $2 \mathrm{n}=24$; (5) Hedypnois ragadioloides, $2 \mathrm{n}=8 ;(6)$ Hypochoeris salzmanniana, $2 \mathrm{n}=8$. Escala $5 \mu \mathrm{m}$. 


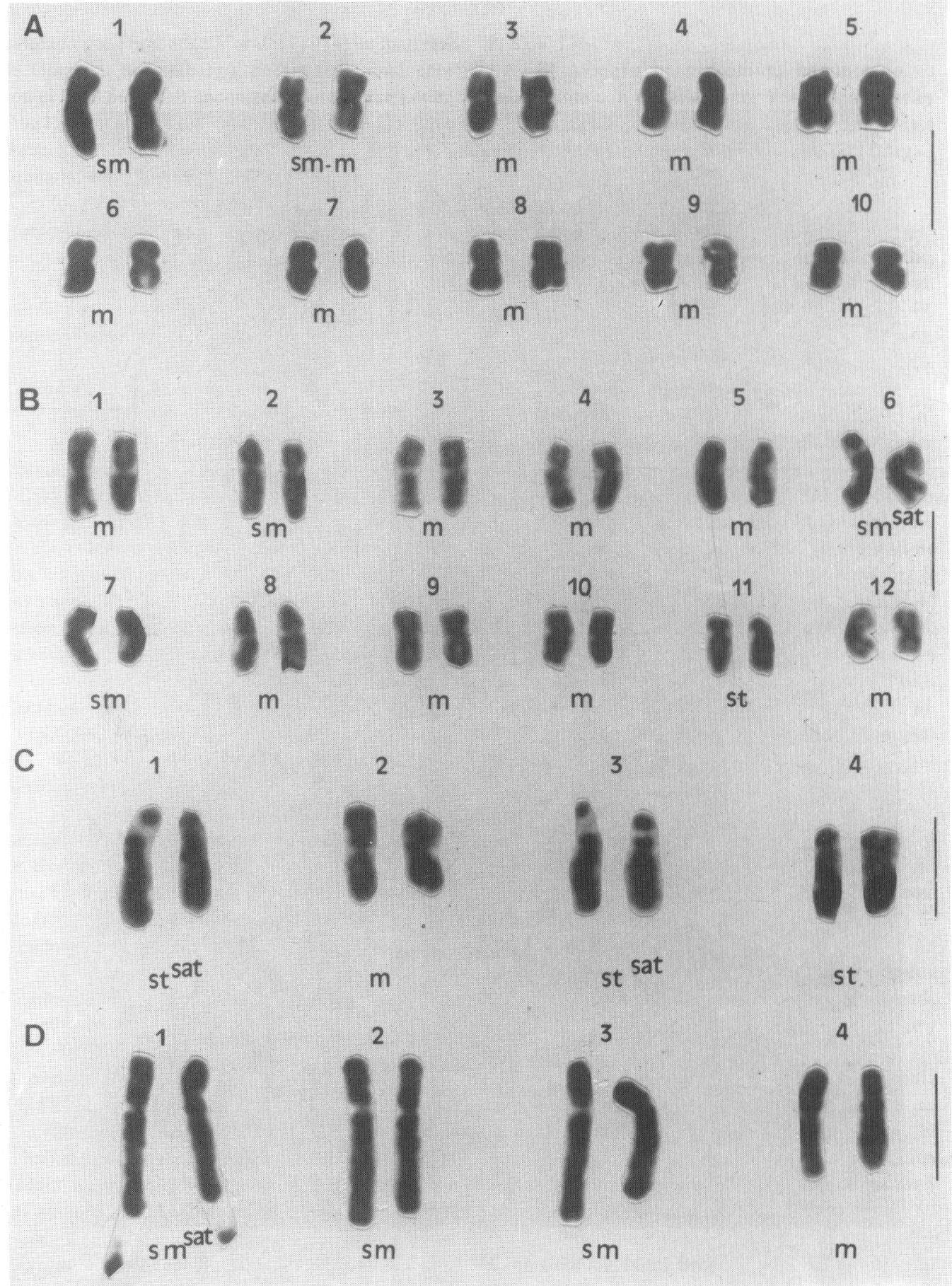

Figura 2. Cariogramas de A, Centaurea bombycina SEV 136153; B, Centaurea sulfurea SEV 136146; C, Hedypnois ragadioloides SEV 136147; D, Hypochoeris salzmanniana SEV 136148. Escala $5 \mu \mathrm{m}$. 
IV.1995, S. Talavera y J. Pavón, 40/1, SEV 136145.

Este número cromosómico coincide con el hallado por Fernandes \& Queirós (1971) en material de Portugal. Valdés-Bermejo y Agudo Mata (1983) encuentran en material de Huelva $2 n=30$ y observan 2 cromosomas supernumerarios en su material. Sin embargo, difiere del $2 n=24$ encontrado por Guinochet \& Foissac (1962) en material también de Portugal. Al parecer es el segundo recuento de esta especie en España.

Hedypnois ragadioloides (L.) F.G. Schmidt $2 \mathrm{n}=8$ (fig. $1.5,2 \mathrm{C}$ )

CÁDIZ: La Línea, arenales costeros. V.1995. Arista, M., P. L. Ortiz y S. Talavera, SEV 136147.

El número cromosómico encontrado coincide con el indicado por Fernandes \& Queiros (1971 y 1972) en material de Portugal así como por el de Capireni et al. (1978) y Bartolo et al. (1978). Sin embargo, difiere de los números $2 \mathrm{n}=10,11,12,13$, 14,16 y 18 encontrados por diversos autores sub $H$. cretica, H. globulifera y H. tubiformis (véase Pastor, 1992).

El cariograma (fig. 2C) tiene la fórmula $1 \mathrm{~m}+1 \mathrm{st}+2 \mathrm{st}^{\mathrm{tat}}$. El tipo de asimetría es $3 \mathrm{~A}$ y el tamaño aparente de los cromosmas en nuestro material osciló entre 4,4-5 $\mu \mathrm{m}$ (medianamente pequeños)

Hypochoeris salzmanniana DC.

$2 \mathrm{n}=8$ (fig. $1.6,2 \mathrm{D})$

CÁDIZ: La Línea, arenales costeros. V.1995. Arista, M., P. L. Ortiz y S. Talavera. SEV 136148.

El número somático encontrado confirma el hallado por Talavera (1981) en material procedente de Cádiz. Al parecer es la segunda vez que se estudia este taxón desde el punto de vista cariológico.

La fórmula cromosómica del cariograma (fig. 2D) es $1 \mathrm{~m}+2 \mathrm{sm}+1 \mathrm{sm}^{\text {sat }}$. El tamaño aparente de los cromosomas osciló entre 4,7-7 $\mu \mathrm{m}$ (medianamente pequeños y medianamente grandes respectivamente). El tipo de asimetría es $4 \mathrm{~A}$.

\section{BIBLIOGRAFÍA}

BARtolo, G., S. BRULlo, M. GRILlo, P. PAVONE \& A. ZIZZA -1978- Numeri cromosomici per la Flora italiana: 382-397. Inform. Bot. Ital. , 10 (1): 64-80.
BLANCA LÓPEZ, G. -1981- Notas cariosistemáticas en el género Centaurea L. Sect. Wilkommia G. Blanca. II. Conclusiones. Anales Jard. Bot. Madrid, 38: 109-125.

CAPIRENI, R., G. D'AMATO \& P. MARCHI 1978- Numeri cromosomici per la Flora italiana: 534-583. Inform. Bot. Ital., 10 (2): 421-465.

CASTROVIEJO, S. y E. VALDÉS-BERMEJO 1991- Archivos de Flora Iberica Núm. 1. Números cromosomáticos de plantas vasculares Ibéricas, I. CSIC. Madrid.

CHIAPPINI, M. -1954- Ricerche sullo sviluppo embriologico di alcune specie del genere Centaurea L. (Asteraceae). Nuovo Giorn. Bot. Ital., 61: 274-289.

FERNANDES, A. \& M. QUEIRÓS -1971Contribution à la connaissance cy totax onomique des Spermatophyta du Portugal. II. Compositae. Bol. Soc. Brot., Sér. 2:, 45: 5-121.

FERNANDES, A. \& M. QUEIRÓS -1972- Systèmes génétiques chez Hedypnois Scop. I. Microsporogénèse. Bol. Soc. Brot., Sér. 2, 46: 5-62.

FERNÁNDEZ MORALES, M.J. -1974- Estudios citogenéticos en especies españolas del género Centaurea. Granada.

GADELLA, TH., E. KLIPHUIS \& A. MENNEGA 1966-Chromosome numbers of some flowering plants of Spain and France. Acta Bot. Neerl., 15: 484-489.

GALLEGO, M.J. y A. APARICIO -1990- Números cromosómicos para la Flora española, 603-617. Lagascalia, 15: 288-295.

GUINOCHET, M. \& J. FOISSAC -1962- Sur les caryotipes de quelques espèces du genre Centaurea L. et leur signification taxonomique. Rev. Cytol. Biol.-Végét., 25: 373.

KHIDIR, M.O. \& P.F. KNOWLES -1970Cytogenetic studies of Carthamus species (Compositae) with 32 pairs of chromosomes. Amer. J. Botany, 57: 123-129.

LEVAN, A., K. FREDGA \& A. SANDBERG -1964Nomenclature for centromeric position on chromosomes. Hereditas, 52: 201-220.

LUQUE, T., J.A. MEJÍAS y Z. DÍAZ LIFANTE 1988- Números cromosómicos para la Flora española, 544-550. Lagascalia, 15: 130-133.

PASTOR, J. -1992-Atlas cromosómico de la Flora vascular de Andalucía Occidental. Universidad de Sevilla. 
SNOW, R. -1963- Alcoholic hydrochloric acidcarmine as a stain for chromosomes in squach preparations. Stain Technol., 38: 9-13.

STEBBINS, G.L. -1938-Cytological characteristics associated with the different growth habits in the dicoyledons. Amer. J. Bot., 25: 189-198.

STEBBINS, G.L. -1971- Chromosomal evolution in higher plants. London.

TALAVERA, S. -1981- Números cromosómicos para la Flora española, 201-205. Lagascalia, 10: 232-238.

TALAVERA, S., J.A. DEVESA y E.F. GALIANO 1984- Notas cariosistemáticas sobre plantas norteafricanas. I. Compositae. Candollea, 39 (1): 271-280.

VALDÉS, B. -1973- Números cromosómicos de algunas plantas españolas. I. Lagascalia, 3: 211217.
VALDÉS-BERMEJO, E. y M.P. AGUDO MATA 1983-Estudios cariológicos en especies Ibéricas del género Centaurea L. (Compositae). Anales J. Bot. Madrid, 38: 119-142.

VALDÉS-BERMEJO, E. -1979- Números cromosomáticos de plantas occidentales, 1-34. Anales J. Bot. Madrid, 36: 373-389.

Aceptado para su publicación en Julio de 1995

Dirección de los autores. Departamento de Biología Vegetal y Ecología. Universidad de Sevilla. Apdo. 1095. 41080 Sevilla. 